\title{
Review: intensive multidisciplinary biopsychosocial rehabilitation reduces pain and improves function in chronic low back pain
}

Guzmán J, Esmail R, Karjalainen K, et al.Multidisciplinary bio-psycho-social rehabilitation for chronic low back pain. Cochrane Database Syst Rev 2002;(1):CD000963 (latest version 12 Oct 2001).

\author{
QUESTION: In patients with chronic disabling low back pain, does multidisciplinary \\ biopsychosocial rehabilitation reduce pain and improve function, employment status, and \\ quality of life?
}

\section{Data sources}

Studies were identified by searching Medline, CINAHL, EMBASE/Excerpta Medica, PsycLIT, HealthSTAR, and the Cochrane Library from database inception to June 1998; reviewing reference lists; and consulting the editors of the Back Review Group of the Cochrane Collaboration.

\section{Study selection}

Studies were selected if they were randomised controlled trials (RCTs) comparing multidisciplinary biopsychosocial rehabilitation with a non-multidisciplinary control intervention in adults with disabling low back pain for $>3$ months. Trials had to report on $\geq 1$ of pain, function, employment status, quality of life, or global improvement. Trials evaluating pure educational interventions or pure physical interventions were excluded.

\section{Data extraction}

Data were extracted by 2 independent reviewers on study quality, patient characteristics, intervention type and duration, and outcomes.

\section{Main results}

10 studies involving 1964 patients met the selection criteria. Study results were not combined in a meta-analysis because of heterogeneity. Evidence was considered to be strong when multiple high quality trials showed consistent findings; moderate when multiple low quality RCTs or 1 high quality and $\geq 1$ low quality RCT produced generally consistent findings; and limited when only 1 RCT reported the finding or if findings across trials were inconsistent. There was strong evidence that intensive multidisciplinary biopsychosocial rehabilitation with a functional restoration approach (ie, a programme designed to improve functional capabilities and includes strength and cardiovascular training, stretching, pacing and planning of daily activities, functional movements, relaxation and management, and coping skills) improved function when compared with inpatient or outpatient non-multidisciplinary treatments. There was moderate evidence that intensive multidisciplinary biopsychosocial rehabilitation with a functional restoration approach reduced pain when compared with outpatient non-multidisciplinary rehabilitation or usual care. There was contradictory evidence regarding the effects of an intensive multidisciplinary biopsychosocial intervention on employment outcomes: 1 trial reported improvements in work readiness, but 2 others showed no reductions in sickness leaves. Less intensive out- patient psychophysical treatments (5 trials) did not improve pain, function, or employment outcomes when compared with non-multidisciplinary outpatient treatment or usual care. Few trials reported effects on quality of life or global assessments.

\section{Conclusions}

In patients with chronic disabling low back pain, intensive multidisciplinary biopsychosocial rehabilitation with a functional restoration approach reduces pain and improves function. Less intensive interventions do not improve clinically relevant outcomes.

\section{COMMENTARY}

Finding an effective way to treat disabling low back pain is of interest to healthcare professionals and patients. This systematic review by Guzmán et al is timely. It includes only RCTs, and thus extends our existing knowledge in this area. The results apply only to patients with severe disability, defined as being unable to work or conduct regular activities of daily life for 3 months.

This systematic review was well done. It seems appropriate that the authors did not do a meta-analysis given the wide range of types of programmes and interventions included in the review. However, the clinical significance of the statistically significant improvement reported in this review was not addressed and remains unclear. Most included trials were done in Scandinavia, and none were done in the US or the UK. It is difficult to tell from the systematic review whether any characteristics of the included trials might limit the generalisability of the finding to other countries. Duration of follow up in the studies varied from immediately after treatment to 5 years. Future studies should ensure adequate follow up because treatment effects may fade by 24 months. Quality of life was rarely assessed in the primary studies and should be included as an outcome in future work.

This review found that intensive programmes with $>100$ hours of treatment were effective, whereas programmes of $<30$ hours were not effective. This suggests that relatively long, multidisciplinary programmes are required. Currently, these types of programmes may not be available in primary care settings. Less intensive treatments did not seem to be effective. As the authors point out, healthcare funders may not be prepared to pay for such a resource, and economic evaluations in this area are urgently needed.

Kate Seers, RGN, PhD Head of Research Royal College of Nursing Institute Radcliffe Infirmary Oxford, UK 